\title{
OPEN Identification of potential therapeutic antimicrobial peptides against Acinetobacter baumannii in a mouse model of pneumonia
}

\author{
Chiau-Jing Jung ${ }^{1 \bowtie}$, You-Di Liao ${ }^{2}$, Chih-Chieh Hsu ${ }^{3}$, Ting-Yu Huang ${ }^{4}$, Yu-Chung Chuang ${ }^{5}$, \\ Jeng-Wei Chen ${ }^{6,7}$, Yu-Min Kuo ${ }^{7}$ \& Jean-San $\mathrm{Chia}^{4,8} \bowtie$
}

Acinetobacter baumannii-induced nosocomial pneumonia has become a serious clinical problem because of high antibiotic resistance rates. Antimicrobial peptides (AMP) are an ideal alternative strategy due to their broad-spectrum of antimicrobial activity and low incidence of bacterial resistance. However, their application is limited by toxicity and stability in vivo. The present study used a mouse model to directly identify potential AMPs effective for treatment of $A$. baumanniiinduced pneumonia. Fifty-eight AMPs were screened and two identified (SMAP-29 and TP4) to have prophylactic effects which prevented the death of mice with pneumonia. Furthermore, two TP4 derivatives ( $\mathrm{dN} 4$ and $\mathrm{dC4}$ ) were found to have therapeutic activity in pneumonia mouse models by peritoneal or intravenous administration. Both dN4 and dC4 also inhibited and/or eliminated $A$. baumannii biofilms at higher doses. Taken together, these data suggest the AMP derivatives dN4 and dC4 represent a potential treatment strategy for $A$. baumannii-induced pneumonia.

Acinetobacter baumannii is a Gram-negative opportunistic pathogen with high incidence among immunocompromised individuals ${ }^{1}$. It largely causes nosocomial and community-acquired pneumonia, skin and urinary tract infections, bacteremia, and surgical site infections $s^{2,3}$. The rapid global emergence of multidrug-resistant A. baumannii (MDRAB) has been a great public health concern recently ${ }^{4,5}$. The World Health Organization has listed antibiotic-resistant $A$. baumannii as a top priority bacterial pathogen that requires extensive research and development of new and effective antimicrobial agents ${ }^{6}$. In addition, A. baumannii is able to form biofilms on the endotracheal surface, which may account for pneumonia onset in patients who require mechanical ventilation ${ }^{3,7}$. These factors substantially contribute to a mortality rate of $35 \%$ or higher in patients with nosocomial A. baumannii infection ${ }^{8,9}$, depending on the patient's condition and bacterial strain involved. Therefore, it is imperative to search for new therapeutic approaches to combat the MDR threat from A. baumannii.

To treat MDR bacterial infections, various novel antimicrobial strategies have been developed, such as antimicrobial peptides [AMPs $]^{10}$. AMPs are ancient components of the innate immune system found across all kingdoms of life that are less likely to trigger drug resistance ${ }^{11}$. These peptides are composed of a varying number of amino acids (from five to $>100$ ) and usually have a broad spectrum of target organisms, ranging from viruses to parasites. In general, AMPs are cationic, targeting bacterial cell membranes and causing disintegration of the lipid bilayer which leads to cytoplasmic leakage and bacterial death ${ }^{12}$. Other antimicrobial mechanisms of action have also been reported ${ }^{13}$, including delocalization of membrane proteins ${ }^{14}$, alteration of cytoplasmic membrane septum formation ${ }^{15}$, as well as inhibition of cell wall ${ }^{16}$, DNA, RNA, and protein synthesis ${ }^{17,18}$ and enzymatic activity ${ }^{13,19}$. In addition to their bactericidal activity, some AMPs also have the ability to kill bacteria inside the biofilm or inhibit biofilm formation directly ${ }^{20}$. Despite these advantageous features, challenges to the

\footnotetext{
${ }^{1}$ Department of Microbiology and Immunology, School of Medicine, College of Medicine, Taipei Medical University, No. 250, Wuxing Street, Taipei 11031, Taiwan. ${ }^{2}$ Institute of Biomedical Sciences, Academia Sinica, Taipei, Taiwan. ${ }^{3}$ Graduate Institute of Oral Biology, School of Dentistry, National Taiwan University, Taipei, Taiwan. ${ }^{4}$ Graduate Institute of Microbiology, College of Medicine, National Taiwan University, No. 1, Jen Ai Road Section 1, Taipei 10051, Taiwan. ${ }^{5}$ Department of Internal Medicine, National Taiwan University Hospital, Taipei, Taiwan. ${ }^{6}$ Division of Cardiovascular Surgery, Department of Surgery, National Taiwan University Hospital, Taipei, Taiwan. ${ }^{7}$ Graduate Institute of Clinical Medicine, College of Medicine, National Taiwan University, Taipei, Taiwan. ${ }^{8} \mathrm{Graduate}$ Institute of Clinical Dentistry, School of Dentistry, National Taiwan University, Taipei,

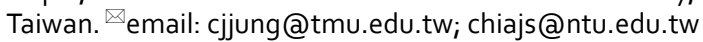




\begin{tabular}{|c|c|c|c|c|c|c|}
\hline \multirow[b]{2}{*}{ Peptide } & \multicolumn{2}{|l|}{17,978} & \multicolumn{2}{|l|}{19,606} & \multicolumn{2}{|c|}{ MDRAB clinical strains\# } \\
\hline & $\operatorname{MIC}(\mu \mathrm{g} / \mathrm{ml})$ & $\operatorname{MBC}(\mu \mathrm{g} / \mathrm{ml})$ & MIC $(\mu \mathrm{g} / \mathrm{ml})$ & $\operatorname{MBC}(\mu \mathrm{g} / \mathrm{ml})$ & $\operatorname{MIC}(\mu \mathrm{g} / \mathrm{ml})$ & $\operatorname{MBC}(\mu \mathrm{g} / \mathrm{ml})$ \\
\hline A2 & 32 & 32 & 8 & 8 & $8-32$ & $8-64$ \\
\hline BMAP-27 & 16 & 16 & 8 & 8 & $4-16$ & $8-32$ \\
\hline CAME & 32 & 32 & 32 & 32 & $8-32$ & $8-64$ \\
\hline SMAP-29 & 8 & 8 & 8 & 8 & $4-32$ & $4-64$ \\
\hline Hla & 32 & 32 & 16 & 16 & $8-16$ & $8-32$ \\
\hline Latarcin 2a & 16 & 16 & 16 & 16 & $8-64$ & $8-64$ \\
\hline MaximinH2 & 64 & 128 & 32 & 32 & $16-128$ & $16-128$ \\
\hline NRC12 & 16 & 16 & 16 & 16 & $8-32$ & $8-32$ \\
\hline Pilosulin & 16 & 32 & 16 & 16 & $8-16$ & $8-32$ \\
\hline Pleurocidin & 16 & 16 & 16 & 16 & $8-32$ & $8-32$ \\
\hline Q6 & 16 & 16 & 16 & 16 & $8-16$ & $8-32$ \\
\hline TP4 & 16 & 16 & 16 & 16 & $8-32$ & $8-32$ \\
\hline
\end{tabular}

Table 1. Minimal inhibitory concentration (MIC) and minimal bactericidal concentration (MBC) of antimicrobial peptides against Acinetobacter baumannii 17,978, 19,606 and multiple drug-resistant (MDR) clinical strains. ${ }^{\#}$ Details are available in Supplementary Table 2.

application of AMPs remain, such as potential toxicity to humans ${ }^{21}$, sensitivity to harsh environmental conditions (e.g., extreme $\mathrm{pH}$ ), and susceptibility to proteases in the circulation ${ }^{22,23}$. Many AMPs work in vitro but fail in vivo. However, because AMPs are composed of amino acids, it is relatively easy to modify their structures in order to lower toxicity and improve stability against proteases ${ }^{24}$. In addition, although previous studies have identified several AMPs exhibiting antimicrobial activity against MDRAB in vitro ${ }^{25,26}$, only a few have tested the effects of AMPs in vivo, especially A. baumannii-induced pneumonia. Therefore, in the present study, we directly used a mouse model of A. baumannii-induced pneumonia to screen for AMPs that can effectively treat MDRAB in vivo. The AMPs having the greatest clinical therapeutic potential were identified in this study.

\section{Results}

In vitro antimicrobial activity of AMPs against laboratory and clinical MDRAB. The antimicrobial activities of 58 AMPs were screened against A. baumannii laboratory strains (ATCC strains 17,978 and 19,606) in vitro using a disc diffusion assay (Supplementary Table 1). Among them, 12 exhibited antimicrobial activity: GW-A2, GW-H1a, GW-Q6 ${ }^{27}$, bovine myeloid antimicrobial peptide-27 [BMAP-27] ${ }^{28}$, cecropin A (1-8)-melittin (1-10) $(\text { CAME })^{29}$, sheep myeloid antimicrobial peptide [SMAP-29] ${ }^{30}$, latarcin $2 \mathrm{a}^{31,32}$, maximin $\mathrm{H} 2^{33}, \mathrm{NRC}_{2}{ }^{34}$, pilosulin ${ }^{35}$, pleurocidin ${ }^{36}$, and $\mathrm{TP} 4^{37}$. Their minimal inhibitory concentration (MIC) and minimal bactericidal concentration (MBC) against A. baumannii laboratory and clinical MDRAB strains were further determined (Table 1 and Supplementary Table 2). Notably, most MDRAB strains exhibited resistance to carbapenem antibiotics, including imipenem and meropenem (Supplementary Table 3) and showed susceptibility to these AMPs (Table 1 and Supplementary Table 1).

In vivo antimicrobial activity of AMPs in A. baumannii-induced murine pneumonia. Murine $A$. baumannii-induced pneumonia models were established by intratracheal inoculation of $A$. baumannii ATCC strain $17978^{38}$. Three different infection doses were tested: $1 \times 10^{9}, 5 \times 10^{8}$, and $1 \times 10^{8}$ colony forming unit (CFU). Intratracheal infection of $1 \times 10^{9}$ and $5 \times 10^{8} \mathrm{CFU}$ doses resulted in death within $4 \mathrm{~d}$ (Supplementary Fig. S1A). Although the $1 \times 10^{8} \mathrm{CFU}$ dose did not cause death, A. baumannii was detected in the lung tissue along with increased infiltration of neutrophils and macrophages (Supplementary Fig. S1B,C,D).

To evaluate the effect of AMPs, an infection dose of $5 \times 10^{8} \mathrm{CFU}$ was used and mouse survival monitored. Since studies have indicated AMPs have cellular toxicity or lose antimicrobial activity in the circulation, the prophylactic effect of prescreened AMPs in vivo was examined by intratracheal administration in pneumonia mouse models. Among the 12 AMPs tested, only SMAP-29 and TP4 significantly prevented the mortality of $A$. baumannii-infected mice (Fig. 1A). Notably, the results of the in vitro time killing assay showed that SMAP-29 and TP4 can rapidly kill $A$. baumannii within 10 min (Fig. 1B).

In vitro antimicrobial activity ofTP4 derivatives against laboratory and clinical MDRAB. SMAP29 and TP4 have high potential for controlling microbial infections $s^{39,40}$. Both exhibit broad-spectrum activity against bacteria and show therapeutic activity in mice. However, their high cellular toxicity and hemolytic activity limit their application. Previous studies have enhanced the application potential of SMAP-29 by modifying its structure $^{41,42}$. Similarly, the hemolytic activity of TP4 was reduced in our previous study by generating truncated and amino acid-substituted analogues ${ }^{43}$. The antimicrobial activities of these derivatives against Pseudomonas aeruginosa, Staphylococcus aureus, and Candida albicans were also investigated in the same previous study ${ }^{43}$. Here, the antimicrobial activity (MIC and MBC) of TP4 derivatives (dN2, dN4, dC2, dC4, F1A/I2A, H3A/ H4A, I5A/I6A, L9A/F10A, I16R, I16E, I16A, L19H/I20H, A12V/A15H, A12I/A15I, R18S/R21H, VHSH, and TP3) was tested in vitro against laboratory and clinical MDRAB. Most of the TP4 derivatives that exhibited 
A
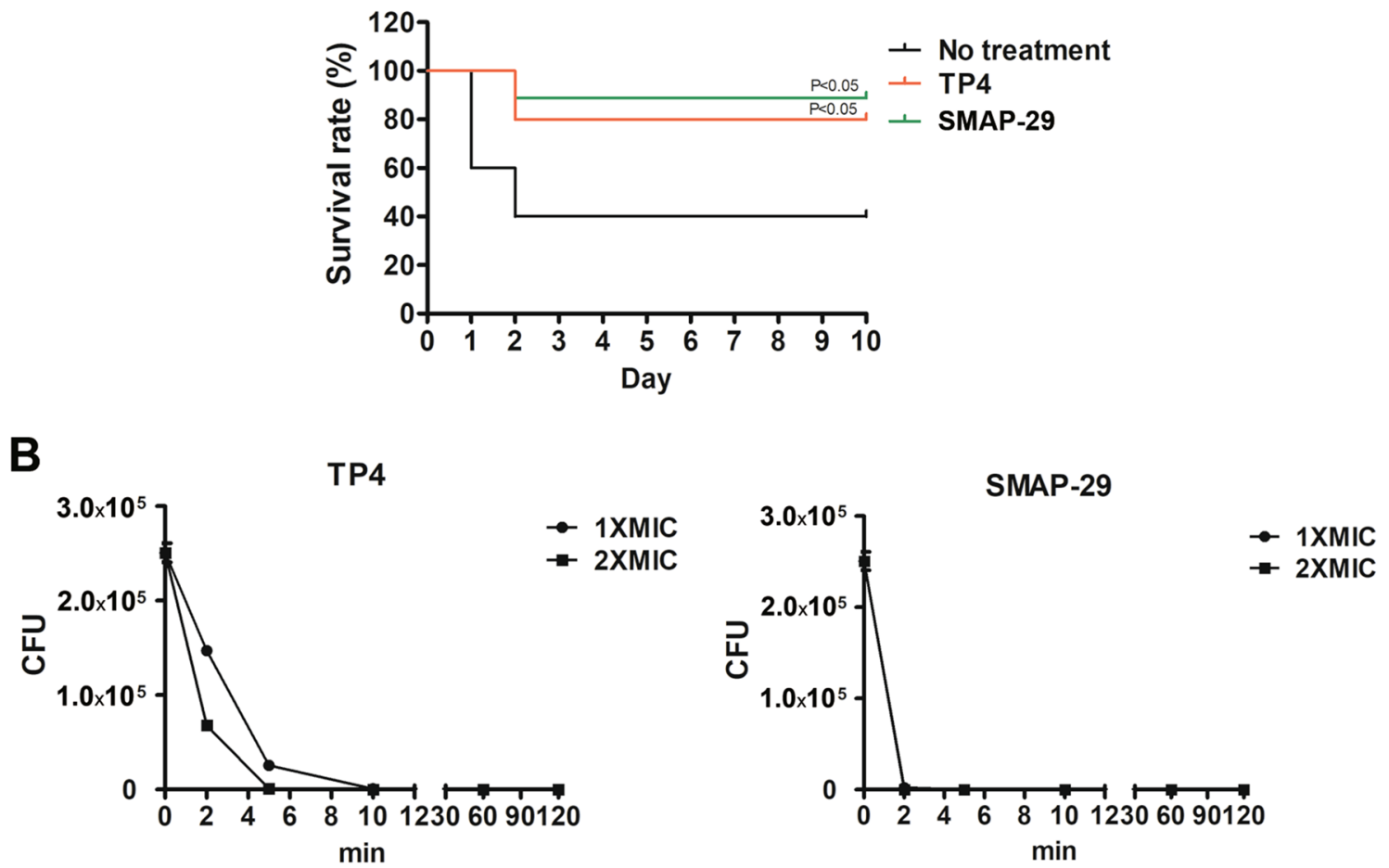

Figure 1. Prophylactic intratracheal administration of TP4 and SMAP-29 reduced mortality associated with $A$. baumannii-induced pneumonia. (A) Mice were intratracheally infected with A. baumannii ATCC 17,978 at a dose of $5 \times 10^{8} \mathrm{CFU}$. A $10-\mu \mathrm{L}$ aliquot of $2 \times$ MIC of TP4 $(16 \mu \mathrm{g} / \mathrm{mL})$ and SMAP-29 $(32 \mu \mathrm{g} / \mathrm{mL})$ was intratracheally administered $30 \mathrm{~min}$ before bacterial infection and mouse survival detected daily ( $n=10 /$ group). (B) A. baumannii ATCC $17,978\left(2-5 \times 10^{5} \mathrm{CFU}\right)$ were mixed with $1 \times$ and $2 \times \mathrm{MIC}$ of TP4 $(8$ and $16 \mu \mathrm{g} / \mathrm{mL})$ or SMAP-29 (16 and $32 \mu \mathrm{g} / \mathrm{mL})$ and incubated at $37^{\circ} \mathrm{C}$. The survival bacteria were detected by plating on agar plate.

reduced hemolytic activity ${ }^{43}$ also showed antimicrobial activity but higher MIC/MBC level against MDRAB strains in vitro (Table 2).

In vivo therapeutic effect of TP4 derivatives in A. baumannii-induced murine pneumonia. Peritoneal administration of TP4 reportedly exhibits a therapeutic effect in S. aureus-induced infection in mice ${ }^{39}$. Therefore, the therapeutic effect of peritoneal administration of TP4 derivatives in A. baumannii-induced pneumonia mouse models was assessed in the present study. Among 17 TP4 derivatives, six (dN4, dC4, H3A/H4A, L9A/F10A, I16R, and R18S/R21H) enhanced the survival rate of pneumonia mouse models (Fig. 2). Notably, $\mathrm{dN} 4$ and $\mathrm{dC} 4$ enhanced survival the most and had the lowest hemolytic activity ${ }^{43}$. In addition, dN4 and dC4 can also rapidly kill bacteria (Fig. 2B). Therefore, the therapeutic effect of intravenous dN4 and dC4 in mice was examined (Fig. 3). Intravenous administration of $\mathrm{dN} 4$ or dC4 $4 \mathrm{~h}$ after inoculation of mice with $\mathrm{A}$. baumannii reduced bacterial numbers inside the lung and enhanced the survival rate (Fig. 3A,B). Administration of dN4 or dC4 once a day for three days enhanced more mouse survival to chi-square significantly enhanced level (Fig. 3C). These data suggest the potential of $\mathrm{dN} 4$ and $\mathrm{dC} 4$ in the clinical control of $A$. baumannii-induced pneumonia.

dN4 and dC4 exhibited antimicrobial activity against $A$. baumannii biofilm. In addition to colonizing lung tissue, $A$. baumannii can form a biofilm on the surface of the endotracheal tube which may account for causing pneumonia in patients who require mechanical ventilation ${ }^{3,7}$. Therefore, the antimicrobial activity of dN4 and dC4 against A. baumannii biofilm were further tested. Both of dN4 and dC4 inhibited A. baumannii biofilm formation in a dose-dependent manner (Fig. 4A) and eliminated established biofilm (Fig. 4B,C). These data suggest that both $\mathrm{dN} 4$ and $\mathrm{dC} 4$ can prevent and/or eliminate A. baumannii biofilm on the surface of the endotracheal tube.

\section{Discussion}

MDR bacteria-induced infection is one of the toughest issues facing clinics today as several are currently untreatable, including MDRAB. Among alternative strategies under development, AMPs are ideal antimicrobial agents because they do not readily trigger resistance. However, application of AMPs is limited by their high cellular toxicity and loss of function in the circulation ${ }^{23}$; hence, many AMPs work in vitro but fail in vivo. Therefore, the 


\begin{tabular}{|c|c|c|c|c|c|c|c|c|}
\hline \multirow[b]{3}{*}{ Peptide } & \multicolumn{4}{|l|}{ ATCC strains } & \multicolumn{4}{|c|}{ MDRAB strains } \\
\hline & \multicolumn{2}{|l|}{\begin{tabular}{|l|}
17,978 \\
\end{tabular}} & \multicolumn{2}{|l|}{19,606} & \multicolumn{2}{|c|}{ BF50 } & \multicolumn{2}{|l|}{ M091 } \\
\hline & MIC $(\mu \mathrm{g} / \mathrm{ml})$ & MBC $(\mu \mathrm{g} / \mathrm{ml})$ & $\operatorname{MIC}(\mu \mathrm{g} / \mathrm{ml})$ & MBC $(\mu \mathrm{g} / \mathrm{ml})$ & MIC $(\mu \mathrm{g} / \mathrm{ml})$ & MBC $(\mu \mathrm{g} / \mathrm{ml})$ & MIC $(\mu \mathrm{g} / \mathrm{ml})$ & $\operatorname{MBC}(\mu \mathrm{g} / \mathrm{ml})$ \\
\hline TP4 & 16 & \begin{tabular}{|l|l}
16 \\
\end{tabular} & 16 & 16 & 16 & \begin{tabular}{|l|l|}
16 \\
\end{tabular} & \begin{tabular}{|l|l}
16 \\
\end{tabular} & 32 \\
\hline $\mathrm{dN} 2$ & 32 & 32 & 64 & 64 & 32 & 64 & 64 & 64 \\
\hline $\mathrm{dN} 4$ & 32 & 32 & 16 & 64 & 16 & 64 & 32 & 32 \\
\hline $\mathrm{dC} 2$ & 16 & 16 & 16 & 32 & 16 & 32 & 16 & 32 \\
\hline $\mathrm{dC} 4$ & 32 & 32 & 32 & 32 & 32 & 32 & 32 & 32 \\
\hline F1A/I2A & 32 & 32 & 16 & 16 & 16 & 32 & 32 & 32 \\
\hline $\mathrm{H} 3 \mathrm{~A} / \mathrm{H} 4 \mathrm{~A}$ & 64 & 128 & 64 & 64 & 64 & 128 & 64 & 256 \\
\hline $\mathrm{I} 5 \mathrm{~A} / \mathrm{I} 6 \mathrm{~A}$ & 32 & 32 & 64 & 64 & 32 & 64 & 64 & 128 \\
\hline L9A/F10A & 64 & 64 & 64 & 128 & 64 & 64 & 128 & 128 \\
\hline I16R & 128 & 128 & 64 & 64 & 64 & 128 & 64 & 256 \\
\hline $\mathrm{I} 16 \mathrm{E}$ & $>256$ & $>256$ & $>256$ & $>256$ & $>256$ & $>256$ & $>256$ & $>256$ \\
\hline I16A & 16 & 32 & 32 & 32 & 16 & 16 & 16 & 32 \\
\hline L19H/I20H & 32 & 32 & 64 & 64 & 32 & 32 & 32 & 32 \\
\hline $\mathrm{A} 12 \mathrm{~V} / \mathrm{A} 15 \mathrm{H}$ & 16 & 32 & 16 & 32 & 16 & 128 & 16 & 256 \\
\hline A12I/A15I & 64 & 64 & 32 & 128 & 64 & 128 & 32 & 128 \\
\hline R18S/R21H & 16 & 32 & 32 & 32 & 16 & 64 & 32 & 32 \\
\hline VHSH & 16 & 64 & 16 & 16 & 32 & 32 & 32 & 64 \\
\hline TP3 & 32 & 32 & 16 & 32 & 32 & 32 & 16 & 32 \\
\hline
\end{tabular}

Table 2. Minimal inhibitory concentration (MIC) and minimal bactericidal concentration (MBC) of antimicrobial peptide TP4 derivatives against Acinetobacter baumannii ATCC and clinical multiple drugresistant (MDR) strains.

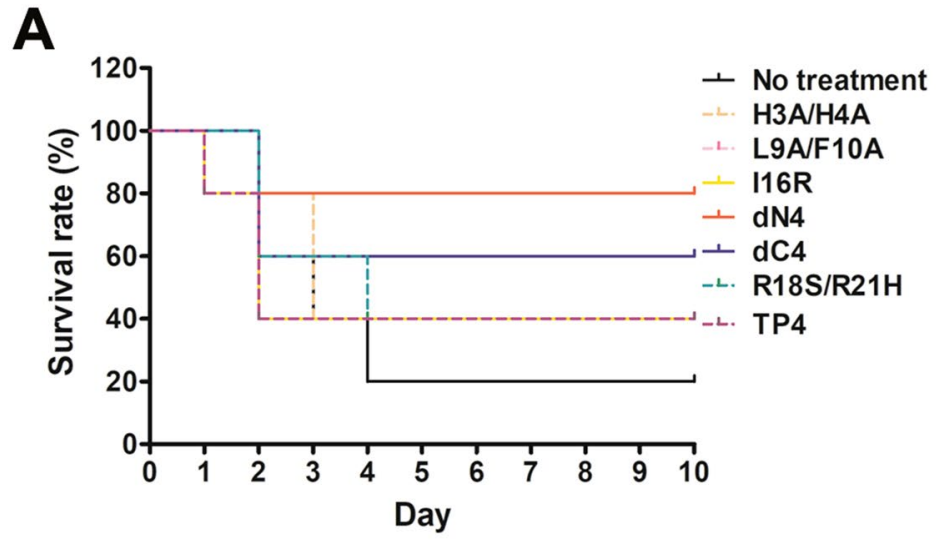

B
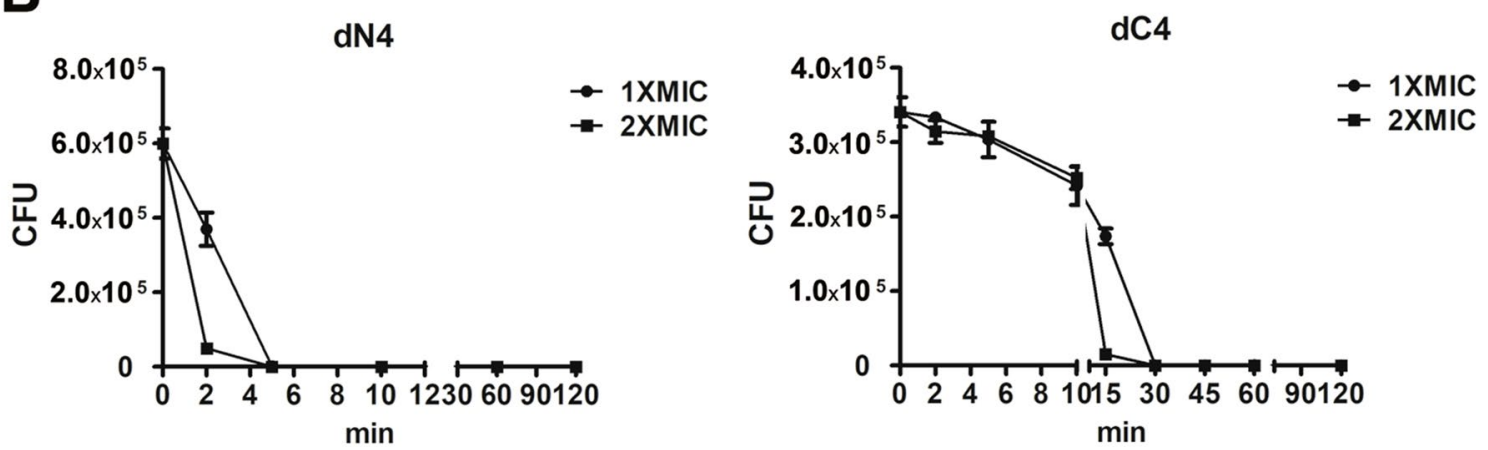

Figure 2. Peritoneal administration of TP4 derivatives in A. baumannii-induced pneumonia mouse models. (A) Mice were intratracheally infected with A. baumannii ATCC 17,978 at a dose of $5 \times 10^{8}$ CFU. TP4 derivatives $(2 \mathrm{mg} / \mathrm{kg})$ were peritoneally administrated $4 \mathrm{~h}$ after bacterial infection, and mouse survival was detected daily ( $n=5$ /group). (B) A. baumannii ATCC 17,978 (2-5 $\left.\times 10^{5} \mathrm{CFU}\right)$ were mixed with $1 \times$ and $2 \times \mathrm{MIC}$ of dN4 (32 and $64 \mu \mathrm{g} / \mathrm{mL})$ or dC4 $(32$ and $64 \mu \mathrm{g} / \mathrm{mL})$, and incubated at $37^{\circ} \mathrm{C}$. The survival bacteria were detected by plating on agar plate. 
A

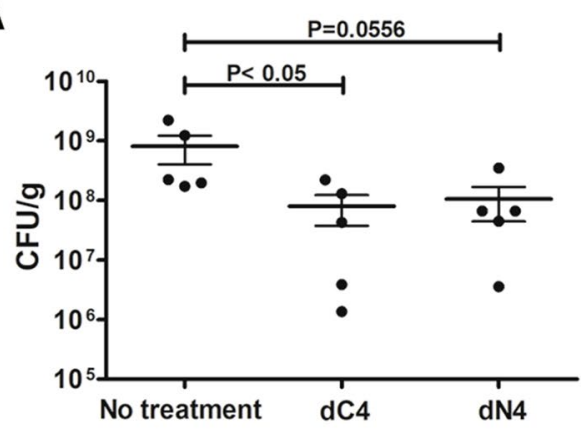

B

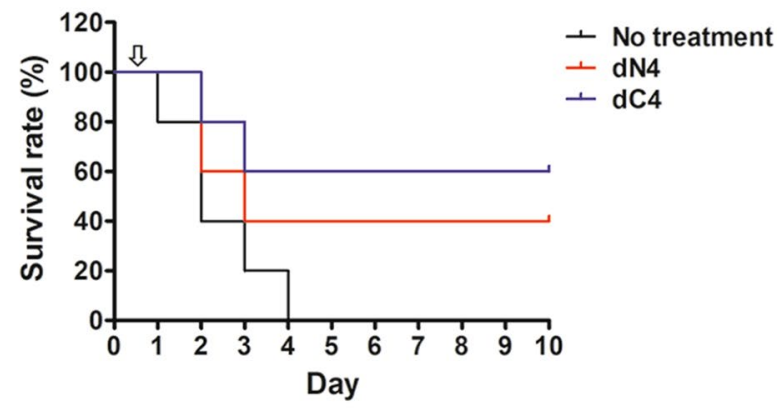

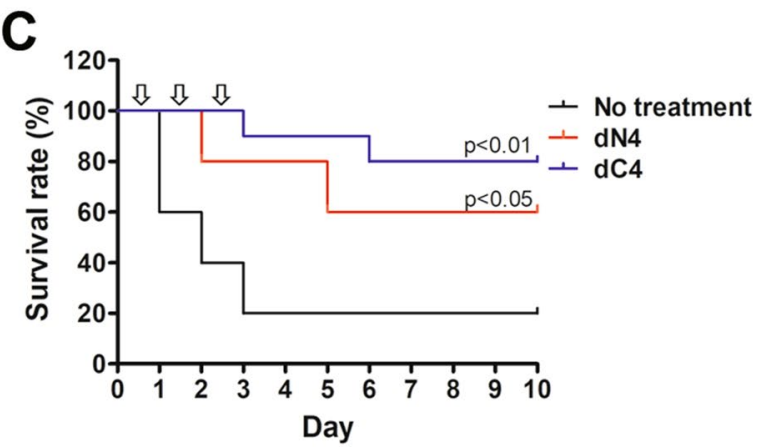

Figure 3. Intravascular administration of $\mathrm{dN} 4$ and $\mathrm{dC} 4$ reduced bacteria numbers in lung tissue and reduced mortality associated with $A$. baumannii-induced pneumonia. (A) Mice were intratracheally infected with $A$. baumannii ATCC 17,978 at a dose of $5 \times 10^{8}$ CFU. dC4 or dN4 $(2 \mathrm{mg} / \mathrm{kg})$ was intravascularly administrated $4 \mathrm{~h}$ after bacterial infection. Infected mice were sacrificed $1 \mathrm{~d}$ after infection, and lung tissue was isolated. Bacteria that colonized lung tissue were quantified by plating on LB agar plates. Each point represents one mouse. Data represent means \pm standard error of the means and were statistically analyzed using Mann-Whitney U-test versus the no treatment group. (B) Mice were intratracheally infected with A. baumannii ATCC 17,978 at a dose of $5 \times 10^{8} \mathrm{CFU}$. dC4 or dN4 $(2 \mathrm{mg} / \mathrm{kg})$ was intravascularly administrated $4 \mathrm{~h}$ after bacterial infection. Mouse survival was detected daily ( $n=5$ /group). (C) Mice were intratracheally infected with $A$. baumannii ATCC 17,978 at a dose of $5 \times 10^{8} \mathrm{CFU}$. dC4 or dN4 $(2 \mathrm{mg} / \mathrm{kg})$ was intravascularly administered once a day for $3 \mathrm{~d}$. Mouse survival was detected daily ( $n=10$ /group).

present study identified AMPs exhibiting potential to treat A. baumannii-induced pneumonia in mice. In particular, intravenous adminstration of TP4 derivatives $\mathrm{dC} 4$ or $\mathrm{dN} 4$ reduced $A$. baumannii conlonization in the lung and enhanced survival, suggesting preservation of antimicrobial activity in the circulation. In fact, dN4 and dC4 maintained, though reduced, antimicrobial activity against $A$. baumannii in the presence of plasma components in vitro (Supplementary Table 4), implying these derivatives may be more resistant to proteases in the plasma relative to other AMPs tested. In addition, our preliminary data showed that the $\mathrm{LD}_{50}$ of SMAP-29 and TP4 is around $10-20 \mathrm{mg} / \mathrm{kg}$ by intratracheal administration and the $\mathrm{LD}_{50}$ of $\mathrm{dN} 4$ and $\mathrm{dC} 4$ is approxmiately $80-90 \mathrm{mg} /$ $\mathrm{kg}$ and $90-100 \mathrm{mg} / \mathrm{kg}$, respectively, by intravenous administration. However, additional large scale animal testing is needed to determine the cellular toxicity and stablity/half-life of $\mathrm{dN} 4$ and $\mathrm{dC} 4$ for pharmaceutical applications. Although intravenous administration of $\mathrm{dN} 4$ or $\mathrm{dC} 4$ enhanced survival, it should be noted that this required daily administration for three days to achieve significant results. On the other hand, the sample number was small as this was a pilot study conducted to identify AMPs with the potential to treat MDRAB in vivo. More studies with a larger sample size and more dose testing should be performed to confirm the clinical potential of dN4 and dC4.

We previously showed that TP4 exhibits broad-spectrum activity against various bacteria, including $P$. aeruginos $a^{43}$. TP4 binds the P. aeruginosa outer membrane target protein OprI through hydrophobic interactions. Mutation of hydrophobic residues in the main helix or deletion of nonstructural coils at N-and C-termini significantly reduced the hemolytic activity of TP4 and reduced the antimicrobial activity against P. aeruginosa $a^{43}$. The current results showed that most TP4 derivatives, which showed reduced hemolytic activity and reduced antimicrobial activity against $P$. aeruginos $a^{43}$, maintained their antimicrobial activity against $A$. baumannii in the present study (Table 2). This suggests the antimicrobial mechanisms of TP4 against $A$. baumannii and $P$. aeruginosa are different. Although TP4 and many of its derivatives have in vitro antimicrobial activity against A. baumannii, only dN4 and dC4 showed clear protection against A. baumannii-induced pneumoniae in vivo (Fig. 2). This may be attributed to the significantly reduced hemolytic activity of $\mathrm{dN} 4$ and dC4 compared with TP4 and other TP4 derivatives ${ }^{43}$. In addition, $\mathrm{dN} 4$ and $\mathrm{dC} 4$ showed comparatively low antimicrobial activity $(32 \mu \mathrm{g} / \mathrm{mL})$ and low in vivo bactericidal effects (Fig. 3A) with a relatively low therapeutic dose, indicating an indirect antimicrobial effect of $\mathrm{dN} 4$ and dC4 in vivo. Moreover, our preliminary data showed that intratracheal administration of $\mathrm{dN} 4$ or $\mathrm{dC} 4$ can only delay death and not enhance survival, which also supports the indirect 
A

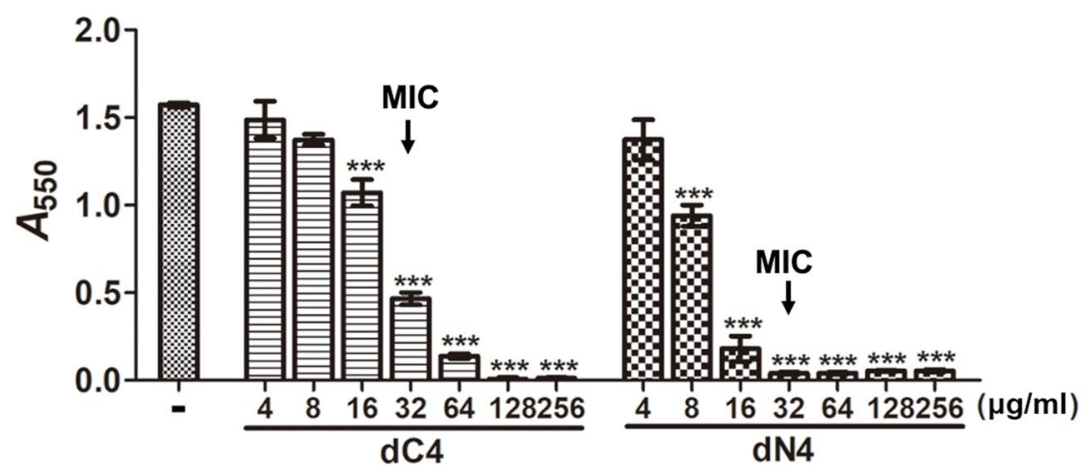

B

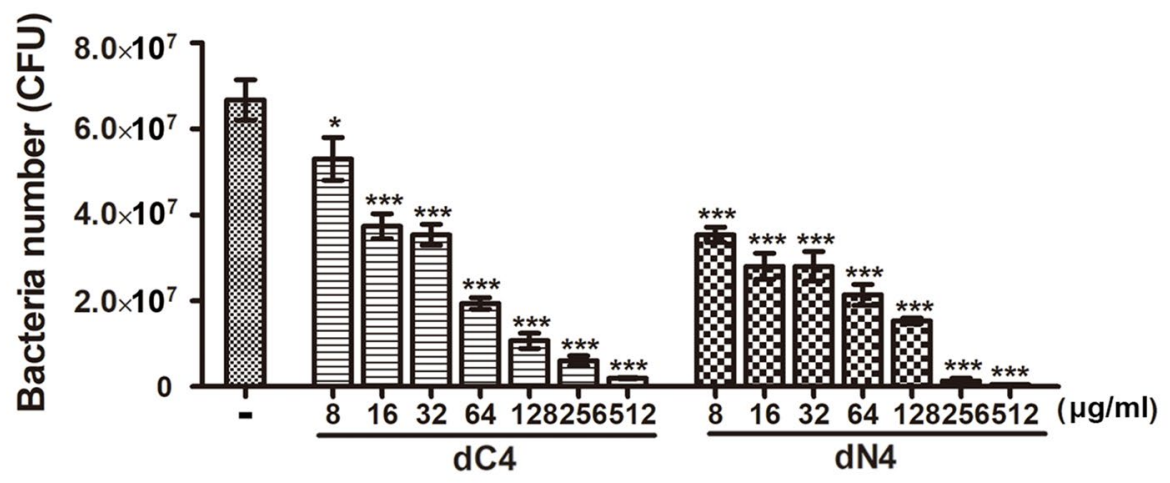

C

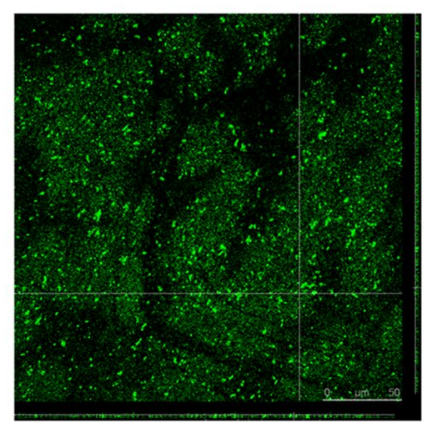

No treatment

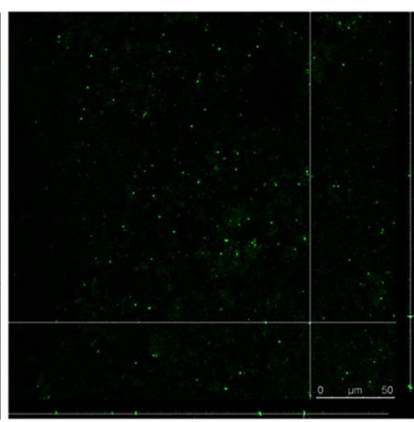

dC4

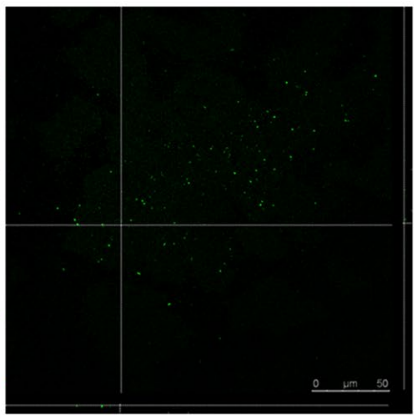

dN4

Figure 4. $\mathrm{dC} 4$ and $\mathrm{dN} 4$ inhibit formation of and eliminate established A. baumannii biofilm. (A) dC4 and dN4 inhibition of A. baumannii biofilm formation. A. baumannii biofilm was grown in LB medium containing serial concentrations of $\mathrm{dC} 4$ or $\mathrm{dN} 4$ and stained with $0.1 \%$ crystal violet. Staining was detected by measuring the absorbance at $550 \mathrm{~nm}$. Data are expressed as the means \pm standard deviations of triplicate experiments; ${ }^{* * *} P<0.001$ by 1 -way analysis of variance. (B) $\mathrm{dC} 4$ and $\mathrm{dN} 4$ elimination of $A$. baumannii biofilm. LB medium containing serial concentrations of $\mathrm{dC} 4$ or $\mathrm{dN} 4$ was added onto formed A. baumannii biofilm. After overnight incubation, the survived bacteria were quantified by plating on LB agar plates. Data are expressed as means \pm standard deviations of triplicate experiments; ${ }^{* * *} P<0.001$ by 1 -way analysis of variance. (C) Confocal laser scanning microscopy images show $\mathrm{dC} 4$ and $\mathrm{dN} 4$ elimination of $A$. baumannii biofilm. LB medium with or without $256 \mu \mathrm{g} / \mathrm{mL}$ of $\mathrm{dC} 4$ or $\mathrm{dN} 4$ was added to established A. baumannii biofilm. A. baumannii were labeled with GFP (green). After overnight incubation, the biofilms were observed by confocal microscope $(400 \times$ magnification) .

antimicrobial activity of $\mathrm{dN} 4$ and $\mathrm{dC} 4$ in the circulation. TP4 has been shown to modulate the host immune response thereby enhancing clearance of Helicobacter pylori infection ${ }^{44}$. Therefore, the indirect antimicrobial effect of $\mathrm{dN} 4$ and dC4 in vivo may be attributed to the immune modulation ability of these AMPs against $A$. baumannii infection, which will require further elucidation.

In addition to colonizing lung tissue, A. baumannii form a biofilm on the surface of the endotracheal tube, accounting for pneumonia onset in patients requiring mechanical ventilation ${ }^{3,7}$. Biofilm-producing A. baumannii clinical isolates have also been shown to be more resistant to most antibiotics compared with 
non-biofilm-producing isolates ${ }^{45}$. Bacterial biofilm is well-organized and dense so as to support bacteria localization and growth on a specific surface and facilitate escape from immune surveillance and antimicrobial molecule attack $^{46}$. Therefore, bacterial biofilms are even more resistant to antibiotic treatment versus platonic populations. Although the current results showed that the A. baumannii biofilm is more resistant to dN4 and dC4 than platonic populations, both derivatives can successfully inhibit $A$. baumannii biofilm formation in vitro (Fig. 4A) as well as eliminate established biofilms and the bacteria residing in them (Fig. 4B,C). These results suggest that $\mathrm{dN} 4$ and $\mathrm{dC} 4$ may be particularly useful for endotracheal tube-associated $A$. baumannii infections. However, the dose of $256 \mu \mathrm{g} / \mathrm{mL}$ was shown to eliminate almost the bacteria inside the biofilm, which is a little high for clinical use. Therefore, use of $\mathrm{dN} 4$ and $\mathrm{dC} 4$ to eradicate established biofilms on an endotracheal tube will require further evaluation.

In summary, the present study identified two AMPs (SMAP-29 and TP4) that exhibited in vitro and in vivo prophylactic effects against $A$. baumannii. Furthermore, TP4 derivatives dC4 and dN4 exhibiting reduced hemolytic activity but high antimicrobial activity against $A$. baumannii both in vitro and in vivo (intravenously and peritoneally). In addition, our preliminary data showed $\mathrm{dN} 4$ and $\mathrm{dC} 4$ also have antimicrobial activity against other bacteria, including antibiotic-resistant pathogens. Considering the extremely limited strategies available to treat MDR pathogen-induced infections, the clinical potential of these AMPs in other MDR pathogen-induced infections should be further evaluated.

\section{Materials and methods}

Bacterial strains and growth conditions. A. baumannii laboratory strains (ATCC 17,978 and 19,606) and clinical isolates from National Taiwan University Hospital (Taipei City, Taiwan) were grown and maintained in Luria-Bertani (LB) broth and agar plates. Medium containing $50 \mu \mathrm{g} / \mathrm{mL}$ kanamycin was used to propagate $A$. baumannii carrying plasmids that expressed green fluorescent protein (GFPuv).

MIC and MBC of AMPs. All methods used in this study were carried out in accordance with relevant guidelines and regulations.

All AMPs and TP4 derivatives used in this study were synthesized by Kelowna International Scientific Inc. (Taipei City, Taiwan) with more than 95\% purity, and their molecular weights were verified by mass spectrum analysis. MBC were determined by extending the MIC procedure in sterilized 96-well polypropylene microtiter plates according to the broth microdilution guidelines of the Clinical and Laboratory Standards Institute with some modification ${ }^{47}$. Briefly, $5 \times 10^{5} \mathrm{CFU} / \mathrm{mL}$ of bacteria were inoculated in LB medium containing AMPs or antibiotic at final concentrations ranging from 512 to $1 \mu \mathrm{g} / \mathrm{mL}$ (from serial twofold dilutions). After incubation for $24 \mathrm{~h}$ at $37^{\circ} \mathrm{C}$, the lowest concentration showing no visible growth was used as the MIC value. In addition, 10 $\mu \mathrm{L}$ was drawn from each well with no visible turbidity onto LB agar plates and incubated for $24 \mathrm{~h}$ at $37^{\circ} \mathrm{C}$. The lowest concentration showing no visible growth on agar subculture was used as the MBC value. All experiments were performed in triplicate.

Experimental A. baumannii-induced pneumonia mouse model. Approval for animal use was obtained from the National Taiwan University Institutional Animal Care and Use Committee prior to initiation of experiments. The study was carried out in compliance with the ARRIVE guidelines (https://arriveguidelines. org). The experimental A. baumannii-induced pneumonia mouse model was generated as previously described with some modification ${ }^{38}$. Male BALB/c mice that were 6-8 weeks-old were purchased from the National Laboratory Animal Center (Nangang Dist, Taipei City, Taiwan). Briefly, $5 \times 10^{8} \mathrm{CFU}$ bacteria were intratracheally inoculated into mice and the survival rate was monitored daily for $10 \mathrm{~d}$. To evaluate prophylactic effects of AMPs, $10 \mu \mathrm{L}$ of $2 \times$ MIC of each AMP was intratracheally administrated to mice 30 min before inoculation of bacteria. For evaluating the therapeutic effect of TP4 derivatives, $2 \mathrm{mg} / \mathrm{kg}$ of each derivative was peritoneally or intravenously administrated $4 \mathrm{~h}$ after bacterial infection or intravenously administrated once a day for three days.

To monitor bacterial colocalization in the lung, mice were sacrificed $1 \mathrm{~d}$ after bacterial infection. Dissected lung tissue was homogenized in a sterile disposable tissue grinder, serially diluted in phosphate-buffered saline (PBS), and plated on LB agar to determine the CFU/g of lung tissue. For histologic analysis, lung tissue was fixed with $2 \%$ paraformaldehyde, embedded in paraffin, cut into $3-\mu \mathrm{m}$-thick slices, and stained with hematoxylin and eosin. Immunohistochemistry was performed using primary antibodies against Ly6G (ab21595; Abcam, Cambridge, MA, USA) or F4/80 and developed with diaminobenzidine (Dako, Santa Clara, CA, USA) followed by counterstaining with Mayer's hematoxylin.

Construction of GFP-tagged $\boldsymbol{A}$. baumannii. For construction of GFPuv-tagged A. baumannii, an A. baumannii GFPuv-expressing plasmid was generated. Briefly, an A. baumannii promoter PgroEL from groEL of A. baumannii was amplified from A. baumannii chromosomal DNA using primers AB_groELPF_XbaI (5' CGA TTATCTAGAGGTGAAGAACTCTTAATTATG-3') and AB_groELPR_KpnI (5'-CGATTAGGTACCCTTGAA ACTTCACGAACAAG-3'). The polymerase chain reaction (PCR) product was digested with KpnI and XbaI and inserted upstream of the gene sequence of GFPuv in the pGFPuv plasmid (Clontech Laboratories, Inc., Mountain View, CA). The GFPuv coding sequence containing the promoter PgroEL was further PCR-amplified using primers AB_groELPF_XbaI and GFPR_XbaI (5'-CGA TTA TCT AGA TTA TTT GTA GAG CTC ATC CAT$3^{\prime}$ ). The PCR product was digested with XbaI and inserted into the E. coli-A. baumannii shuttle plasmid pAbYm3. The resulting plasmid, pABYm3-GFPuv, was transformed into A. baumannii, and positive clones were selected by green fluorescence following ultraviolet excitation $(365 \mathrm{~nm})$. 
Biofilm formation assay. Bacterial biofilm assay was performed as previous described ${ }^{48}$ with modification. Briefly, approximately $10^{7} \mathrm{CFU}$ bacteria in $200 \mu \mathrm{L}$ of LB medium was added to individual wells of a 96-well polystyrene microtiter plate. To investigate the inhibitory effect of AMPs on biofilm formation, serial concentrations of AMPs were added to the culture medium when inoculating bacteria. After a 16-h incubation at $37^{\circ} \mathrm{C}$, the medium was removed, and biofilms were detected by crystal violet staining assay ${ }^{48}$. Each assay was performed in triplicate, and wells without biofilms were used as blank controls. To investigate the effect of AMPs on established biofilm, the biofilms were grown in LB medium at $37^{\circ} \mathrm{C}$ for $16 \mathrm{~h}$. After removing the LB medium containing the planktonic populations of bacteria, the biofilms were washed with sterilized PBS three times, and the medium containing serial concentrations of AMPs was added. After a $16 \mathrm{~h}$ incubation at $37^{\circ} \mathrm{C}$, survival bacteria inside the biofilm were detected by plating on LB medium. For observation by confocal microscopy, the bacteria were transformed with pPABYm3-GFPuv. Bacterial biofilms were grown in 24-well plates with round glass coverslips. The biofilms with or without treatment with AMPs were observed using a confocal microscope (Leica TCS SP5).

Statistical analysis. To analyze the statistical significance of differences between more than two sets of data, 1-way analysis of variance followed by Bonferroni multiple-comparison test was assessed. For analyzing nonparametrically distributed data, a Mann-Whitney U-test was used. Survival curves were compared with logrank tests. A $P<0.05$ was considered statistically significant.

Received: 31 August 2020; Accepted: 18 March 2021

Published online: 01 April 2021

\section{References}

1. Towner, K. J. Acinetobacter: an old friend, but a new enemy. J. Hosp. Infect. 73, 355-363. https://doi.org/10.1016/j.jhin.2009.03. 032 (2009).

2. Peleg, A. Y., Seifert, H. \& Paterson, D. L. Acinetobacter baumannii: emergence of a successful pathogen. Clin. Microbiol. Rev. 21, 538-582. https://doi.org/10.1128/CMR.00058-07 (2008).

3. McConnell, M. J., Actis, L. \& Pachon, J. Acinetobacter baumannii: human infections, factors contributing to pathogenesis and animal models. FEMS Microbiol. Rev. 37, 130-155. https://doi.org/10.1111/j.1574-6976.2012.00344.x (2013).

4. Clark, N. M., Zhanel, G. G. \& Lynch, J. P. 3rd. Emergence of antimicrobial resistance among Acinetobacter species: a global threat. Curr. Opin. Crit. Care 22, 491-499. https://doi.org/10.1097/MCC.0000000000000337 (2016).

5. Gordon, N. C. \& Wareham, D. W. Multidrug-resistant Acinetobacter baumannii: mechanisms of virulence and resistance. Int. J. Antimicrob. Agents 35, 219-226. https://doi.org/10.1016/j.ijantimicag.2009.10.024 (2010).

6. Shlaes, D. M. \& Bradford, P. A. Antibiotics-from there to where?: How the antibiotic miracle is threatened by resistance and a broken market and what we can do about it. Pathog. Immun. 3, 19-43. https://doi.org/10.20411/pai.v3i1.231 (2018).

7. Lee, C. R. et al. Biology of Acinetobacter baumannii: pathogenesis, antibiotic resistance mechanisms, and prospective treatment options. Front. Cell Infect. Microbiol. 7, 55. https://doi.org/10.3389/fcimb.2017.00055 (2017).

8. Antunes, L. C., Visca, P. \& Towner, K. J. Acinetobacter baumannii: evolution of a global pathogen. Pathog. Dis. 71, 292-301. https:// doi.org/10.1111/2049-632X.12125 (2014).

9. Joly-Guillou, M. L. Clinical impact and pathogenicity of Acinetobacter. Clin. Microbiol. Infect. 11, 868-873. https://doi.org/10. $1111 /$ j.1469-0691.2005.01227.x (2005).

10. Ageitos, J. M., Sanchez-Perez, A., Calo-Mata, P. \& Villa, T. G. Antimicrobial peptides (AMPs): ancient compounds that represent novel weapons in the fight against bacteria. Biochem. Pharmacol. 133, 117-138. https://doi.org/10.1016/j.bcp.2016.09.018 (2017).

11. Zasloff, M. Antimicrobial peptides of multicellular organisms. Nature 415, 389-395. https://doi.org/10.1038/415389a (2002).

12. Epand, R. M. \& Vogel, H. J. Diversity of antimicrobial peptides and their mechanisms of action. Biochim. Biophys. Acta 1462, 11-28. https://doi.org/10.1016/s0005-2736(99)00198-4 (1999).

13. Brogden, K. A. Antimicrobial peptides: pore formers or metabolic inhibitors in bacteria?. Nat. Rev. Microbiol. 3, 238-250. https:// doi.org/10.1038/nrmicro1098 (2005).

14. Wenzel, M. et al. Small cationic antimicrobial peptides delocalize peripheral membrane proteins. Proc. Natl. Acad. Sci. USA 111, E1409-1418. https://doi.org/10.1073/pnas.1319900111 (2014).

15. Friedrich, C. L., Moyles, D., Beveridge, T. J. \& Hancock, R. E. Antibacterial action of structurally diverse cationic peptides on gram-positive bacteria. Antimicrob. Agents Chemother. 44, 2086-2092. https://doi.org/10.1128/aac.44.8.2086-2092.2000 (2000).

16. Brotz, H., Bierbaum, G., Leopold, K., Reynolds, P. E. \& Sahl, H. G. The lantibiotic mersacidin inhibits peptidoglycan synthesis by targeting lipid II. Antimicrob. Agents Chemother. 42, 154-160 (1998).

17. Patrzykat, A., Friedrich, C. L., Zhang, L., Mendoza, V. \& Hancock, R. E. Sublethal concentrations of pleurocidin-derived antimicrobial peptides inhibit macromolecular synthesis in E. coli. Antimicrob. Agents Chemother. 46, 605-614. https://doi.org/10.1128/ aac.46.3.605-614.2002 (2002).

18. Mardirossian, M. et al. The host antimicrobial peptide Bac71-35 binds to bacterial ribosomal proteins and inhibits protein synthesis. Chem. Biol. 21, 1639-1647. https://doi.org/10.1016/j.chembiol.2014.10.009 (2014).

19. Otvos, L. Jr. et al. Interaction between heat shock proteins and antimicrobial peptides. Biochemistry 39, 14150-14159. https://doi. org/10.1021/bi0012843(2000).

20. Liu, Z. et al. Tuning the membrane selectivity of antimicrobial peptides by using multivalent design. Chembiochem Eur. J. Chem. Biol. 8, 2063-2065. https://doi.org/10.1002/cbic.200700502 (2007).

21. Matsuzaki, K. Control of cell selectivity of antimicrobial peptides. Biochem. Biophys. Acta. 1788, 1687-1692. https://doi.org/10. 1016/j.bbamem.2008.09.013 (2009).

22. Sieprawska-Lupa, M. et al. Degradation of human antimicrobial peptide LL-37 by Staphylococcus aureus-derived proteinases. Antimicrob. Agents Chemother. 48, 4673-4679. https://doi.org/10.1128/AAC.48.12.4673-4679.2004 (2004).

23. Vlieghe, P., Lisowski, V., Martinez, J. \& Khrestchatisky, M. Synthetic therapeutic peptides: science and market. Drug Discov. Today 15, 40-56. https://doi.org/10.1016/j.drudis.2009.10.009 (2010).

24. Papo, N., Oren, Z., Pag, U., Sahl, H. G. \& Shai, Y. The consequence of sequence alteration of an amphipathic alpha-helical antimicrobial peptide and its diastereomers. J. Biol. Chem. 277, 33913-33921. https://doi.org/10.1074/jbc.M204928200 (2002). 
25. Jaskiewicz, M., Neubauer, D., Kazor, K., Bartoszewska, S. \& Kamysz, W. Antimicrobial activity of selected antimicrobial peptides against planktonic culture and biofilm of Acinetobacter baumannii. Probio. Antimicrob. Proteins 11, 317-324. https://doi.org/10. 1007/s12602-018-9444-5 (2019).

26. Vila-Farres, X. et al. In vitro activity of several antimicrobial peptides against colistin-susceptible and colistin-resistant Acinetobacter baumannii. Clin. Microbiol. Infect. 18, 383-387. https://doi.org/10.1111/j.1469-0691.2011.03581.x (2012).

27. Chou, H. T. et al. Design and synthesis of cationic antimicrobial peptides with improved activity and selectivity against Vibrio spp. Int. J. Antimicrob. Agents 32, 130-138. https://doi.org/10.1016/j.ijantimicag.2008.04.003 (2008).

28. Skerlavaj, B. et al. Biological characterization of two novel cathelicidin-derived peptides and identification of structural requirements for their antimicrobial and cell lytic activities. J. Biol. Chem. 271, 28375-28381 (1996).

29. Wang, Y. Q. \& Cai, J. Y. High-level expression of acidic partner-mediated antimicrobial peptide from tandem genes in E. coli. Appl. Biochem. Biotechnol. 141, 203-213. https://doi.org/10.1007/bf02729062 (2007).

30. Brogden, K. A. et al. The ovine cathelicidin SMAP29 kills ovine respiratory pathogens in vitro and in an ovine model of pulmonary infection. Antimicrob. Agents Chemother. 45, 331-334. https://doi.org/10.1128/AAC.45.1.331-334.2001 (2001).

31. Kozlov, S. A. et al. Latarcins, antimicrobial and cytolytic peptides from the venom of the spider Lachesana tarabaevi (Zodariidae) that exemplify biomolecular diversity. J. Biol. Chem. 281, 20983-20992. https://doi.org/10.1074/jbc.M602168200 (2006).

32. Shlyapnikov, Y. M., Andreev, Y. A., Kozlov, S. A., Vassilevski, A. A. \& Grishin, E. V. Bacterial production of latarcin 2a, a potent antimicrobial peptide from spider venom. Protein Expr. Purif. 60, 89-95. https://doi.org/10.1016/j.pep.2008.03.011 (2008).

33. Lai, R. et al. Antimicrobial peptides from skin secretions of Chinese red belly toad Bombina maxima. Peptides 23, 427-435. https:// doi.org/10.1016/s0196-9781(01)00641-6 (2002).

34. Patrzykat, A., Gallant, J. W., Seo, J. K., Pytyck, J. \& Douglas, S. E. Novel antimicrobial peptides derived from flatfish genes. Antimicrob. Agents Chemother. 47, 2464-2470. https://doi.org/10.1128/aac.47.8.2464-2470.2003 (2003).

35. Zelezetsky, I., Pag, U., Antcheva, N., Sahl, H. G. \& Tossi, A. Identification and optimization of an antimicrobial peptide from the ant venom toxin pilosulin. Arch. Biochem. Biophys. 434, 358-364. https://doi.org/10.1016/j.abb.2004.11.006 (2005).

36. Tao, R. et al. Antimicrobial and antibiofilm activity of pleurocidin against cariogenic microorganisms. Peptides 32, 1748-1754. https://doi.org/10.1016/j.peptides.2011.06.008 (2011).

37. Peng, K. C. et al. Five different piscidins from Nile tilapia, Oreochromis niloticus: analysis of their expressions and biological functions. PLoS ONE 7, e50263. https://doi.org/10.1371/journal.pone.0050263 (2012).

38. Harris, G. et al. A mouse model of Acinetobacter baumannii-associated pneumonia using a clinically isolated hypervirulent strain. Antimicrob. Agents Chemother. 57, 3601-3613. https://doi.org/10.1128/AAC.00944-13 (2013).

39. Huang, H. N., Chan, Y. L., Wu, C. J. \& Chen, J. Y. Tilapia Piscidin 4 (TP4) stimulates cell proliferation and wound closure in MRSAinfected wounds in mice. Mar. Drugs 13, 2813-2833. https://doi.org/10.3390/md13052813 (2015).

40. Dawson, R. M. \& Liu, C.-Q. Cathelicidin peptide SMAP-29: comprehensive review of its properties and potential as a novel class of antibiotics. Drug Dev. Res. 70, 481-498. https://doi.org/10.1002/ddr.20329 (2009).

41. Shin, S. Y. et al. Structure-activity analysis of SMAP-29, a sheep leukocytes-derived antimicrobial peptide. Biochem. Biophys. Res. Commun. 285, 1046-1051. https://doi.org/10.1006/bbrc.2001.5280 (2001).

42. Kalfa, V. C. et al. Congeners of SMAP29 kill ovine pathogens and induce ultrastructural damage in bacterial cells. Antimicrob. Agents Chemother. 45, 3256-3261. https://doi.org/10.1128/AAC.45.11.3256-3261.2001 (2001).

43. Chang, T. W. et al. Hydrophobic residues are critical for the helix-forming, hemolytic and bactericidal activities of amphipathic antimicrobial peptide TP4. PLoS ONE 12, e0186442. https://doi.org/10.1371/journal.pone.0186442 (2017).

44. Narayana, J. L., Huang, H. N., Wu, C. J. \& Chen, J. Y. Efficacy of the antimicrobial peptide TP4 against Helicobacter pylori infection: in vitro membrane perturbation via micellization and in vivo suppression of host immune responses in a mouse model. Oncotarget 6, 12936-12954. https://doi.org/10.18632/oncotarget.4101 (2015).

45. Gurung, J. et al. Association of biofilm production with multidrug resistance among clinical isolates of Acinetobacter baumannii and Pseudomonas aeruginosa from intensive care unit. Indian J. Crit. Care Med. 17, 214-218. https://doi.org/10.4103/0972-5229. 118416 (2013).

46. Stewart, P. S. Mechanisms of antibiotic resistance in bacterial biofilms. Int. J. Med. Microbiol. 292, 107-113. https://doi.org/10. 1078/1438-4221-00196 (2002).

47. National Committee for Clinical Laboratory Standards. Methods for dilution antimicrobial susceptibility tests for bacteria that grow aerobically; Approved Standard, 6th edition. M7-A6. NCCLS, Wayne, PA, 2003.

48. Jung, C. J., Hsu, R. B., Shun, C. T., Hsu, C. C. \& Chia, J. S. AtlA mediates extracellular DNA release, which contributes to Streptococcus mutans biofilm formation in an experimental rat model of infective endocarditis. Infection and immunity 85, doi:https:// doi.org/10.1128/IAI.00252-17 (2017).

\section{Acknowledgments}

This study was supported by Taipei Medical University (TMU105-AE1-B27) and the Ministry of Science and Technology (MOST 108-2320-B-038-057-MY3 and MOST 109-2320-B-002-053).

\section{Author contributions}

C.J.J., Y.D.L., and J.S.C. conceived the experiments, C.C.H., T.Y.H., and J.W.C. developed and conducted the experiments, Y.C.C. provided the clinical MDRAB strains and performed the antibiotic susceptibility testing, Y.M.K. helped to analyze the results, and C.J.J. and J.S.C. wrote the manuscript.

\section{Competing interests}

The authors declare no competing interests.

\section{Additional information}

Supplementary Information The online version contains supplementary material available at https://doi.org/ 10.1038/s41598-021-86844-5.

Correspondence and requests for materials should be addressed to C.-J.J. or J.-S.C.

Reprints and permissions information is available at www.nature.com/reprints.

Publisher's note Springer Nature remains neutral with regard to jurisdictional claims in published maps and institutional affiliations. 
(c) (i) Open Access This article is licensed under a Creative Commons Attribution 4.0 International cc) License, which permits use, sharing, adaptation, distribution and reproduction in any medium or format, as long as you give appropriate credit to the original author(s) and the source, provide a link to the Creative Commons licence, and indicate if changes were made. The images or other third party material in this article are included in the article's Creative Commons licence, unless indicated otherwise in a credit line to the material. If material is not included in the article's Creative Commons licence and your intended use is not permitted by statutory regulation or exceeds the permitted use, you will need to obtain permission directly from the copyright holder. To view a copy of this licence, visit http://creativecommons.org/licenses/by/4.0/.

(C) The Author(s) 2021 